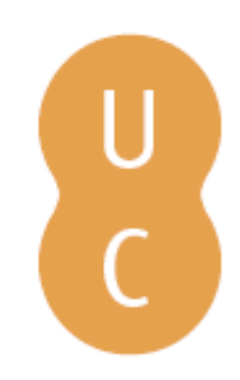

\title{
pompalina
}

\section{O papel da análise de imagem na Geologia Planetária}
Autor(es):
Barata, Teresa; Machado, Adriane
Publicado por: Imprensa da Universidade de Coimbra; Laboratório de Radioactividade Natural da Universidade de Coimbra

URL

persistente:

URI:http://hdl.handle.net/10316.2/36295

DOI:

DOI:http://dx.doi.org/10.14195/978-989-26-1009-2_8

Accessed : $\quad$ 26-Apr-2023 06:38:16

A navegação consulta e descarregamento dos títulos inseridos nas Bibliotecas Digitais UC Digitalis, UC Pombalina e UC Impactum, pressupõem a aceitação plena e sem reservas dos Termos e Condições de Uso destas Bibliotecas Digitais, disponíveis em https://digitalis.uc.pt/pt-pt/termos.

Conforme exposto nos referidos Termos e Condições de Uso, o descarregamento de títulos de acesso restrito requer uma licença válida de autorização devendo o utilizador aceder ao(s) documento(s) a partir de um endereço de IP da instituição detentora da supramencionada licença.

Ao utilizador é apenas permitido o descarregamento para uso pessoal, pelo que o emprego do(s) título(s) descarregado(s) para outro fim, designadamente comercial, carece de autorização do respetivo autor ou editor da obra.

Na medida em que todas as obras da UC Digitalis se encontram protegidas pelo Código do Direito de Autor e Direitos Conexos e demais legislação aplicável, toda a cópia, parcial ou total, deste documento, nos casos em que é legalmente admitida, deverá conter ou fazer-se acompanhar por este aviso. 


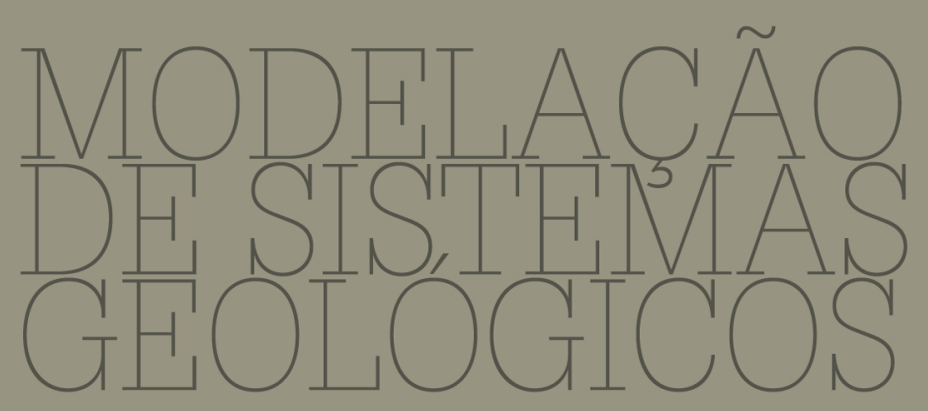

Homenagem ao Professor Doutor Manuel Maria Godinho

L.J.P.F. Neves, A.J.S.C. Pereira,

C.S.R. Gomes, L.C.G. Pereira,

A.O. TAVARES

IMPRENSA DA UNIVERSIDADE DE COIMBRA

CoImBra University Press 


\title{
MODELAÇÃO \\ DE SISTEMAS \\ GEOLÓGICOS
}

Homenagem ao Professor Manuel Maria Godinho

\section{O papel da análise de imagem na Geologia Planetária}

\author{
Teresa Barata ${ }^{1}$; Adriane Machado ${ }^{1}$ \\ ${ }^{1}$ CGUC, Coimbra, PORTUGAL, mtbarata@gmail.com; adrianemachado@yahoo.com.br
}

Palavras-chave: Análise de imagem, Vénus, Estruturas geológicas

\section{Resumo}

Pretende-se, com este trabalho, salientar a importância da análise de imagem na deteç̧ão automática de estruturas geológicas em superfícies planetárias. Apresentam-se alguns exemplos da aplicação de algoritmos de análise de imagem baseados na morfologia matemática, para detectar de uma forma automática, algumas estruturas da superfície de Vénus.

Key-words: Image analysis, Venus, Geological structures

\section{Abstract}

The present work is focused on the importance of image analysis on automatic recognition of geological structures on planetary surfaces. Same examples of image analysis algorithms based on mathematical morphology to automatically detect some structures on Venus surface are shown.

\section{Introdução}

Compreender os planetas telúricos e as luas do nosso Sistema Solar é crucial para a compreender como a Terra se formou e em que direcção irá evoluir. Cada planeta telúrico tem a sua própria história e perceber a natureza e o tempo dos processos geológicos é importante para perceber o seu interior e superfície (Solomon, 2006). Este conhecimento revela-se também essencial para compreender quais as forças que criaram a Terra e que continuam a agir. Contudo, os geólogos planetários não podem actuar como geólogos de campo, tendo que recorrer à interpretação da informação obtida por sistemas de detecção remota e estabelecer comparação com os fenómenos terrestres, mais facilmente acessíveis. Por outras palavras, o estudo de estruturas geológicas de superfícies planetárias, bem como a da sua génese, está 
fortemente baseado na análise de análogos terrestres, tendo por base o pressuposto que estruturas e paisagens similares terão essencialmente a mesma origem.

Nas últimas décadas, novas missões com instrumentos de alta precisão têm visitado os planetas telúricos e adquirido colecções de dados que têm que ser processados e interpretados para que se possa compreender as similaridades e discrepâncias entre planetas. Tradicionalmente a análise de superfícies planetárias baseia-se na interpretação de imagens de detecção remota, processo moroso e sujeito ao "olhar experiente" do analisador. A única excepção é a Terra, em que têm sido desenvolvidas e implementadas técnicas de processamento de imagens com aplicações em várias áreas de investigação, sendo validadas com trabalhos de campo e integradas com outros níveis de informação, recorrendo a Sistemas de Informação Geográfica. Nos últimos anos, baseados em modelos conceptuais de processamento de imagens da Terra, algumas técnicas têm sido desenvolvidas e aplicadas a imagens planetárias, para identificar e caracterizar estruturas comuns tais como terrenos poligonais (Pina et al. 2010; Saraiva et al., 2009), falhas e grabens (Alves et al., 2008; Barata et al., 2009), crateras de impacto e mantos de dejecção (Bandeira et al., 2007; Barata et al., 2010) e campos de dunas (Bandeira et al., 2009, Saraiva et al., 2004).

\section{Análise de imagem}

O desenvolvimento de técnicas computacionais permitiu a consolidação da análise de imagem como uma teoria fundamental que está interessada no aspecto espacial dos objectos em estudo, quaisquer que eles sejam (Serra, 1982). E essa teoria diz-se quantitativa não só por extrair algo que se torna acessível à medida, mas também por lidar com imagens digitais, ou seja, com imagens discretas constituídas por elementos bem identificados espectral e espacialmente. Quer isto dizer que o recurso da análise de imagem em geologia permite não só o reconhecimento automático de estruturas geológicas e a caracterização quantitativa destes fenómenos (parâmetros de forma, sinuosidade, comprimento, anisotropias, entre outros), como também gerar modelos quantitativos e integrá-los com outro tipo de informação.

São vários os métodos de análise de imagem e atendendo a que uma explicação detalhada das diferentes abordagens não se insere no âmbito deste trabalho, serão apenas referidos de uma forma sucinta. Assim, os métodos lineares são reversíveis e baseiam-se na utilização de operadores 
lineares, derivando da teoria de processamento de sinal, filtragem linear (convolução), análise de Fourier. Já os métodos de análise e tratamento de dados recorrem a descritores numéricos susceptíveis de serem agrupados, a métodos estatísticos multivariados (análise em componentes principais, análise factorial de correspondências, análise grupal) de forma a estabelecer uma taxinomia de imagens. Os métodos sintácticos baseiam-se na decomposição da imagem num conjunto de primitivas com significado (discos, segmentos de recta, arcos de contorno), associando regras gramaticais e relações estruturais à sua sucessão. Por último, os métodos morfológicos derivam da teoria de morfologia matemática, introduzida por Matheron e Serra em 1964, que por sua vez se baseia, entre outras, na teoria de conjuntos, e consistem em transformações sequenciais e irreversíveis de imagem, que levam a uma individualização de estruturas filtradas sequencialmente.

Apresentam-se em seguida exemplos de análise de imagem baseada em transformações morfológicas, como objectivo detectar de uma forma automática, estruturas geológicas na superfície planetária de Vénus.

\section{Exemplos de aplicação a Vénus.}

A superfície de Vénus começou a ser sistematicamente observada e visitada na década de sessenta do século passado, quer a partir de observações terrestres, quer a partir de missões espaciais. No entanto, só em 1990, com a missão Magalhães, é que se obteve uma cobertura global do planeta, com resolução suficiente para permitir inferências geológicas. Esta missão permitiu obter imagens de radar com diferentes resoluções espaciais, para além de dados de gravimetria e altimetria.

A superfície de Vénus é jovem, com poucas crateras de impacto, composta por vastas planícies cobertas de lava, devido aos seus vulcões (alguns com caldeiras gigantescas, da ordem dos $100 \mathrm{~km}$ de diâmetro) e montanhas deformadas por actividade geológica. As estruturas tectónicas mais abundantes em Vénus são as wrinkle ridges, estruturas morfologicamente complexas, lineares a sinuosas, resultantes de processos de tensão compressiva, associados a dobramentos e fracturação com encurtamento crustal. Existem ainda estruturas únicas, provavelmente devido a processos de deformação da crusta intensivos, como as tesserae, coronae e aracnóides.

Algoritmos baseados em transformações morfológicas têm vindo a ser desenvolvidos, de forma a reconhecer automaticamente algumas destas estruturas. As figuras seguintes Ilustram exemplos da aplicação destes 
algoritmos às imagens Magalhães de Vénus. Na Figura 1 pode-se observar a imagem original, que abrange uma planície típica de Vénus, onde estão presentes wrinkle ridges e respectivo resultado automático da sua detecção pode ser observado na Figura 2. As direcções principais encontram-se representadas na rosa de direcções da Figura 3.

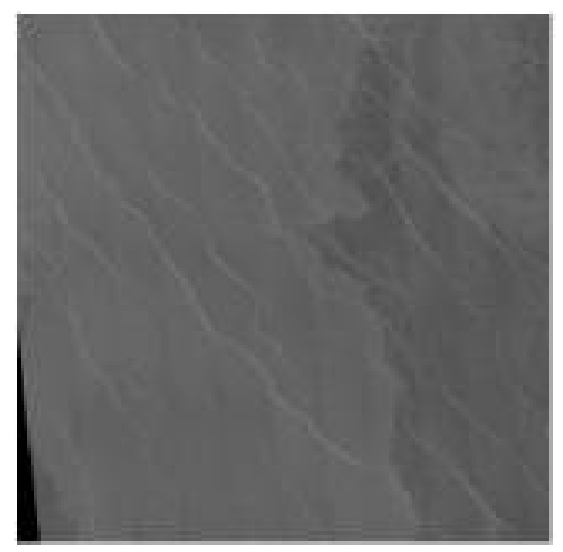

Figura 1. a) Imagem SAR da missão Magalhães de Rusalka Planitia (C1-MIDR.00N180, framelet 19.

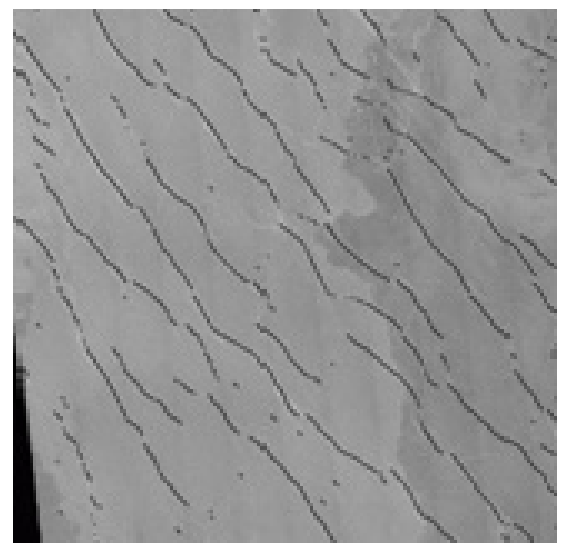

(a)

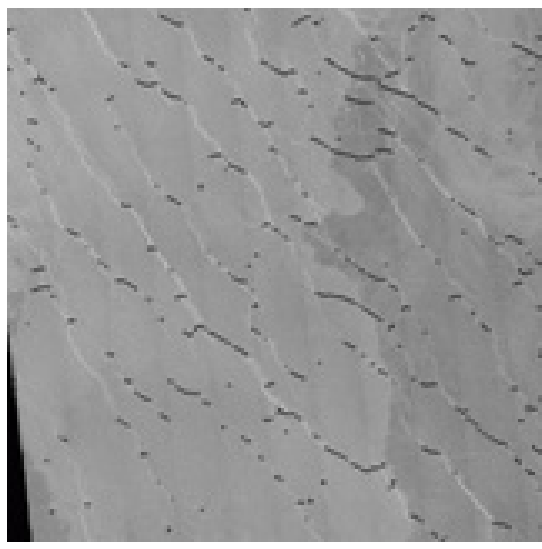

(b)

Figura 2. a) Wrinkle ridges nas direcção: $135^{\circ}$; b) Wrinkle ridges na direcção $0^{\circ}$. 


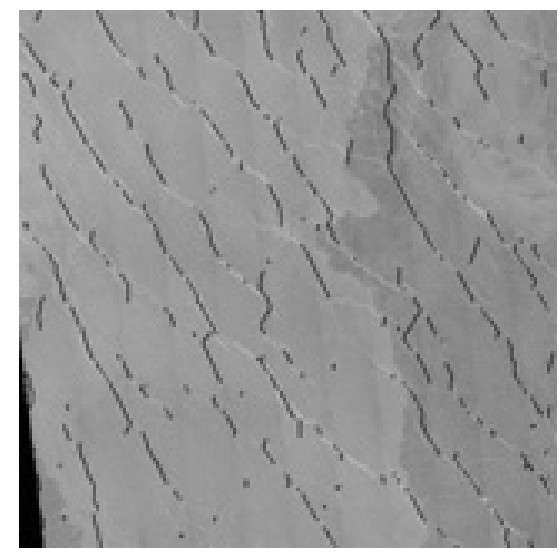

(c)

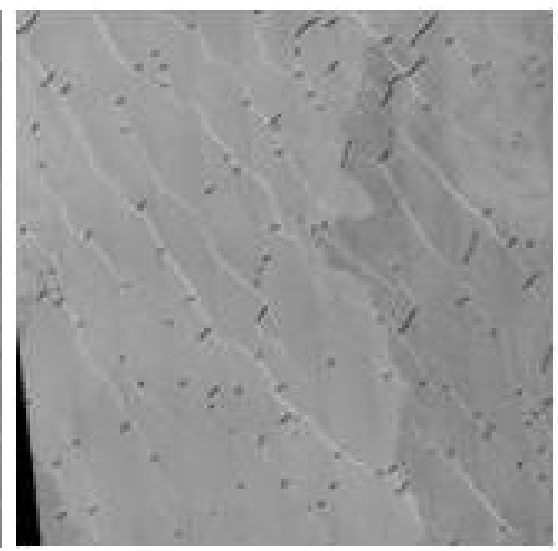

(d)

Figura 2 (cont.). c) Wrinkle ridges na direcção $90^{\circ}$ e d) Wrinkle ridges na direcção $45^{\circ}$.

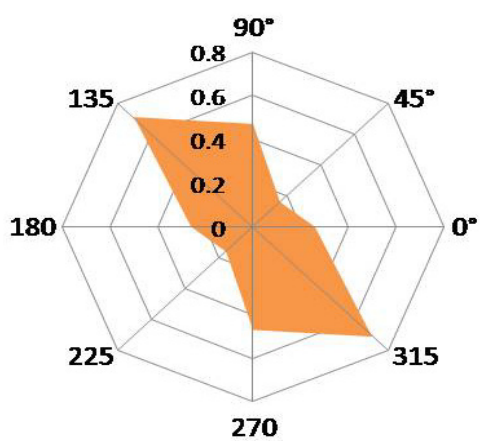

Figura 3. Rosa de direcções obtidas para a imagem de Rusalka Planitia.

Um outro exemplo de algoritmos de detecção automática está ilustrado na próxima figura (4), onde foi identificada uma cratera e os seus depósitos de ejecção. 


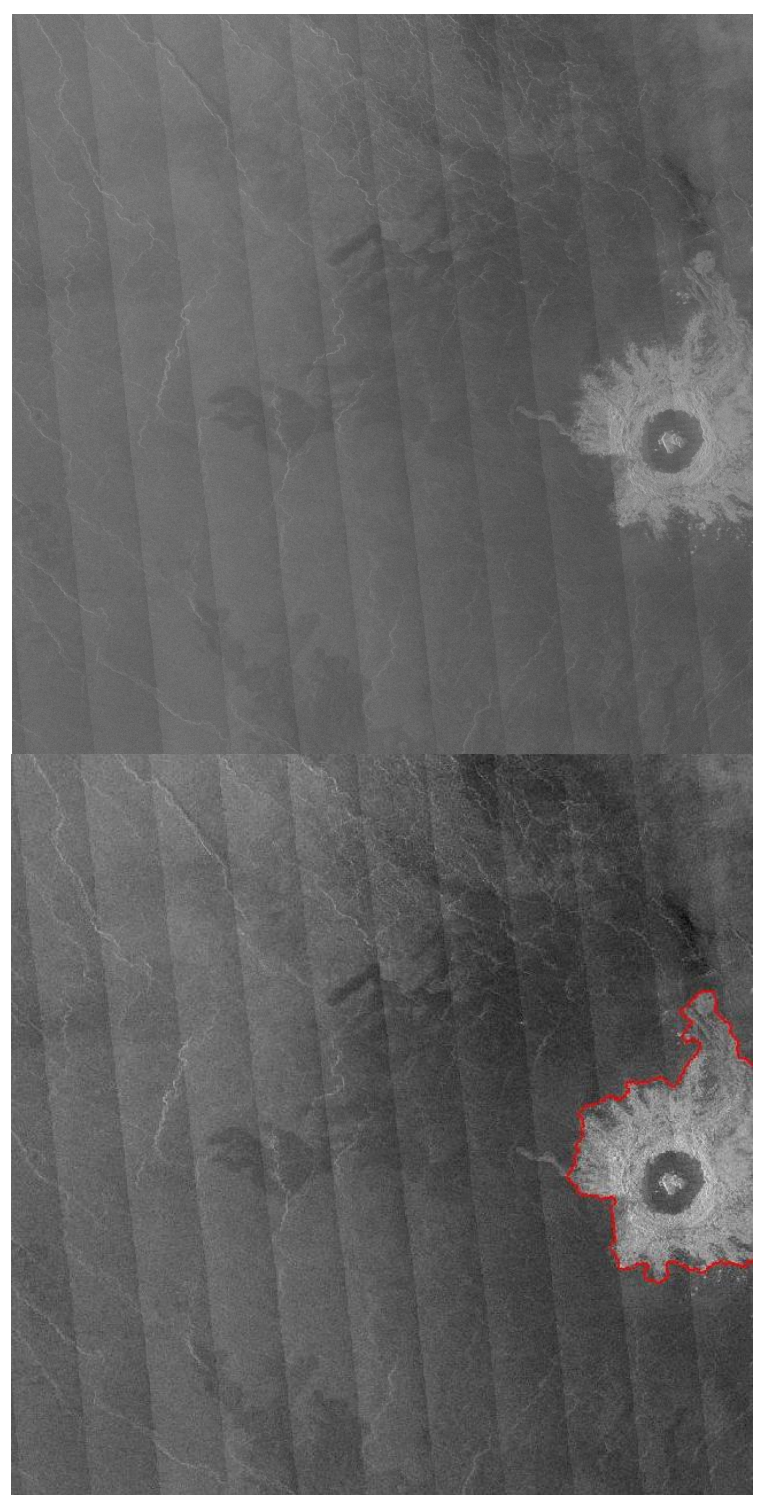

Figura 4. Imagem Magalhães (C1-MIDR.00N180, framelet 24) de Atla Regio, Guinevere Planitia. A imagem mostra a cratera Fossey com depósitos de ejecção; b) detecção automática da cratera Fossey e os seus depósitos de ejecção.

Por último, um exemplo da identificação automática de tesserae está representado na Figura 5. 


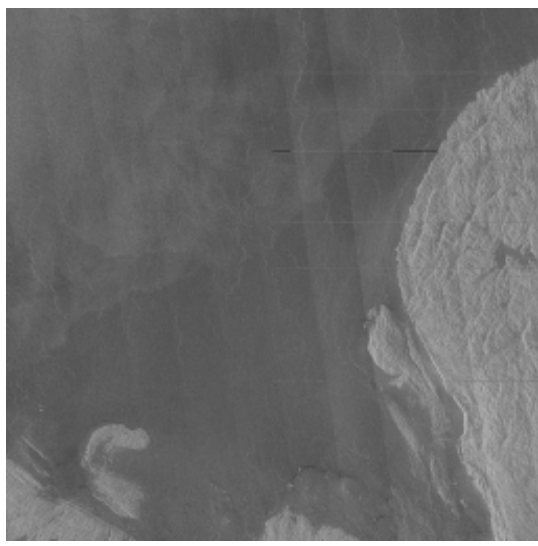

(a)

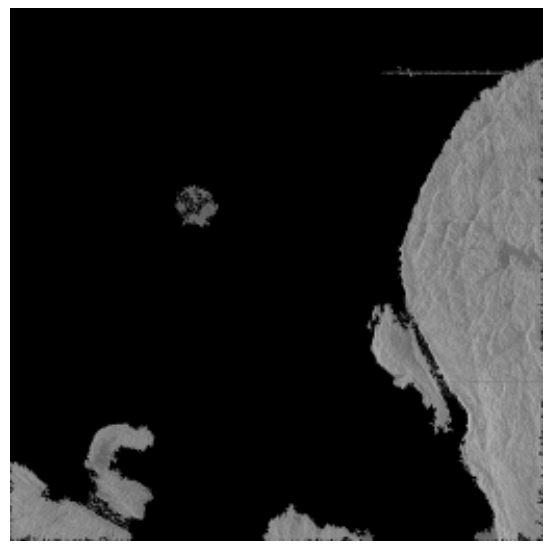

(b)

Figura 5. Imagem Magalhães C1 (a), com a identificação automática de tesserae (b).

\section{Conclusão}

A análise de imagem, teoria que engloba diversas abordagens e técnicas, assume um papel primordial no reconhecimento automático de estruturas geologias de superfícies planetárias, como se pode constatar pelos exemplos apresentados. Esta teoria apresenta a grande vantagem de poder ser aplicada a qualquer imagem de detecção remota. Salienta-se ainda que o reconhecimento automático de estruturas geológicas permite quantificar as suas características, tal como efectuar de uma forma mais expedita a cartografia geológica.

\section{Bibliografia}

Alves E. I., Barata M. T., Vaz D. A., Pereira L. C. G., Baptista A. R., Chorro M. J. (2008) - Martian Tectonics, Water and Life. Lorenzo A. Costas (eds.), Planet Mars Research Focus. Nova Science Publishers, Hauppauge NY; 44pp.

Bandeira 1., Saraiva J., Pina P. (2007) - Impact Crater Recognition on Mars based on probability volume created by template matching. IEEE Transactions on Geoscience and Remote Sensing; 45, 4008-4015.

Bandeira 1. Marques J. e Pina (2009) - Automatic delimitation of regions with aeolian features on Mars. Lunar and Planetary Science XXXX, Abs \# 1025, Lunar and Planetary Institute, Houston, USA.

Barata M. T., Alves E. I., Vaz D. (2009) - Automatic Extraction of Wrinkle Ridges in Venus Magellan Imagery. Lunar and Planetary Science XXXX, Abs \#1025, Lunar and Planetary Institute, Houston, USA.

Barata M. T., Pina P., Saraiva J., Alves E. I. (2010) - Extraction of craters and eject from Venus SAR imagery. Lunar and Planetary Science XXXXI, Abs \# 1412, Lunar and Planetary Institute, Houston, USA. 
Pina P., Vieira G., Christiansen H. H., Barata M. T., Saraiva J., Bandeira L., Lira C. (2010) - Analysis of polygonal terrains on mars based on Svalbard analogues. Lunar and Planetary Science XXXXI, Abs \# 1372, Lunar and Planetary Institute, Houston, USA.

Saraiva J., Bandeira L., Antunes J., Pina P., Barata T. (2009) - Polygonal terrains on Mars. Finisterra, XLIV, 87, 71- 80.

Saraiva J., Pina P., Barata T. (2004) - Automatic recognition of aeolian ripples on Mars. in Lunar and Planetary Science XXXV, Abs \#1621, 2 pp, CD-ROM, Lunar and Planetary Institute, Houston, Texas, USA.

Serra J., (1982) - Image Analysis and Mathematical Morphology - Vol. I, Academic Press, London: $610 \mathrm{p}$.

Solomon, S. C. (2006) - Tectonics on the terrestrial planets. Philadelphia Annual Meeting, paper $\mathrm{n}^{\circ}$ $32-7$. 